\title{
SOBRE LA EXISTENCIA DE POLYPODIUM MACARONESICUM BOBROV EN EL S. DE LA PENINSULA IBERICA
}

\author{
B. DIEZ GARRETAS \& A. E. SALVO-TIERRA *
}

\section{RESUMEN:}

Se cita por primera vez para Europa continental P. macaronesicum Bobrov. Se hace una breve descripción, indicando su corología, ecología, así como su posible origen. Por último se propone una clave para la determinación de las especies europeas del género Polypodium.

\section{SUMMARY:}

P. macaronesicum Bobrov is reported as new to the Flora of continental Europe. Its description, chorological distribution and ecology are discussed. A key to the European species of Polypodium is given.

\section{INTRODUCCION}

El género Polypodium está en la actualidad representado en Europa, por tres especies, que aunque de difícil diágnostico, poseen una buena base genética, $P$. australe Fée, diploide, $2 \mathrm{n}=74$, es el taxon más meridional en Europa con un área de repartición fundamentalmente mediterránea, si bien se extiende por el Atlántico hasta las Islas Británicas; $P$. vulgare L. alotetraploide, $2 \mathrm{n}=148$ extendido por toda Europa y finalmente, $P$. interjectum Shivas, hexaploide, $2 \mathrm{n}=222$, con una distribución europea aún no bien conocida.

Junto a estas tres especies, en el archipiélago macaronésico, se han considerado los táxones, $P$. macaronesicum Bobrov para las Canarias y Madeira y P. azoricum (Vasc.) Fernandes para las Azores.

(*) Departamento de Botánica. Facultad de Ciencias. Universidad de Málaga. 
Recientemente Nardi (1977), ha asimilado P. macaronesicum a $P$. azoricum basándose en la identidad de sus caracteres, al mismo tiempo que subordinaba $P$. azoricum al grupo $P$. australe s.l. considerándolo a nivel subespecífico ( $P$. australe Fée subsp. azoricum (Vasc.) Nardi) admitiendo sin embargo la existencia de biotipos diferentes para cada uno de los archipiélagos macaronésicos, Canarias, Azores y Madeira.

Como resultado de nuestros estudios, no consideramos válida la concepción de Nardi de subordinar $P$. azoricum a $P$. australe y ello en base a que la comparación de caracteres entre ambos táxones presenta las suficientes diferencias como para considerarlas especies distintas (Cuadro I).

Si nos parece en cambio acertada la propuesta de este mismo autor al sinonimizar P. azoricum a P. macaronesicum; por ello y en razón al principio de prioridad nomenclatural el nombre válido sería el propuesto por Bobrov (1964), P. macaronesicum.

\section{CUADRO I}

\section{P. australe \\ P. macaronesicum}

\begin{tabular}{ll}
\hline & $\begin{array}{l}\text { Bien desarrollados } \\
\text { numerosos y ramifi- } \\
\text { cados, más largos } \\
\text { que el esporangio } \\
\text { cuando está maduro. }\end{array}$
\end{tabular}

Poco desarrollados, frágiles y raros, menos ramificados y bastante más cortos que el esporagio maduro y después siempre escondidos en el interior del soros.

Número de células $\quad$ Valor medio indivi-

engrosadas del anillo dual (5)-6-8-(9) de dehiscencia
Valor medio individual, mayor (del orden de $P$. vulgare europeo): (10-) 13-14
Número de células
basales del esporan-
gio

Páleas del rizoma.
Elevado: (2-) 3-4

Más reducido

(del orden $P$. vulgare europeo): 0-1 (-2).
Margen con dientes cortos y distanciados, a veces casi entero.
Margen, más o me- nos denticulado-es- pinoso al menos en las páleas internas y en la parte distal; a veces mas distancia- damente denticulado sobre todo en las es- camas externas.


En Andalucía, hasta el momento presente, se ha podido constatar la presencia de $P$. australe en todas las provincias andaluzas; $P$. vulgare en la de Granada (Salvo Tierra, 1979) y P. interjectum en la de Málaga (Asensi et Diez Garretas, 1975: 7-24).

Recientemente, revisando el material para la Flora Pteridológica de Andalucía, hemos podido comprobar la existencia entre los pliegos depositados en el Herbario de la Facultad de Ciencias de la Universidad de Málaga (MGC) de especímenes cuyos caracteres morfológicos se identificaban con los de P.macaronesicum y que fueron recolectados en diversas localidades de las Sierras de Algeciras en 1973.

Para confirmar esta hipótesis, hemos estudiado un isotipo del material que sirvió de base para la determinación de Bobrov (E. Bourgeau, Plantae canariensis, exs. n. ${ }^{\circ} 189$ ) así como un topotipo del que utilizó Fernandes para la descripción de $P$. azoricum (Ilha do Faial, praia do Norte, 15-I-1963, J. Botelho Gonçalves, LISI 836) del que se conserva un pliego depositado en MGC, n. ${ }^{\circ} 697$.

Tras el estudio y comparación de este material, admitimos la presencia de $P$. macaronesicum en el SW de la Península Ibérica, lo cual representa la primera cita de este taxon para la Flora Pteridológica europea continental.

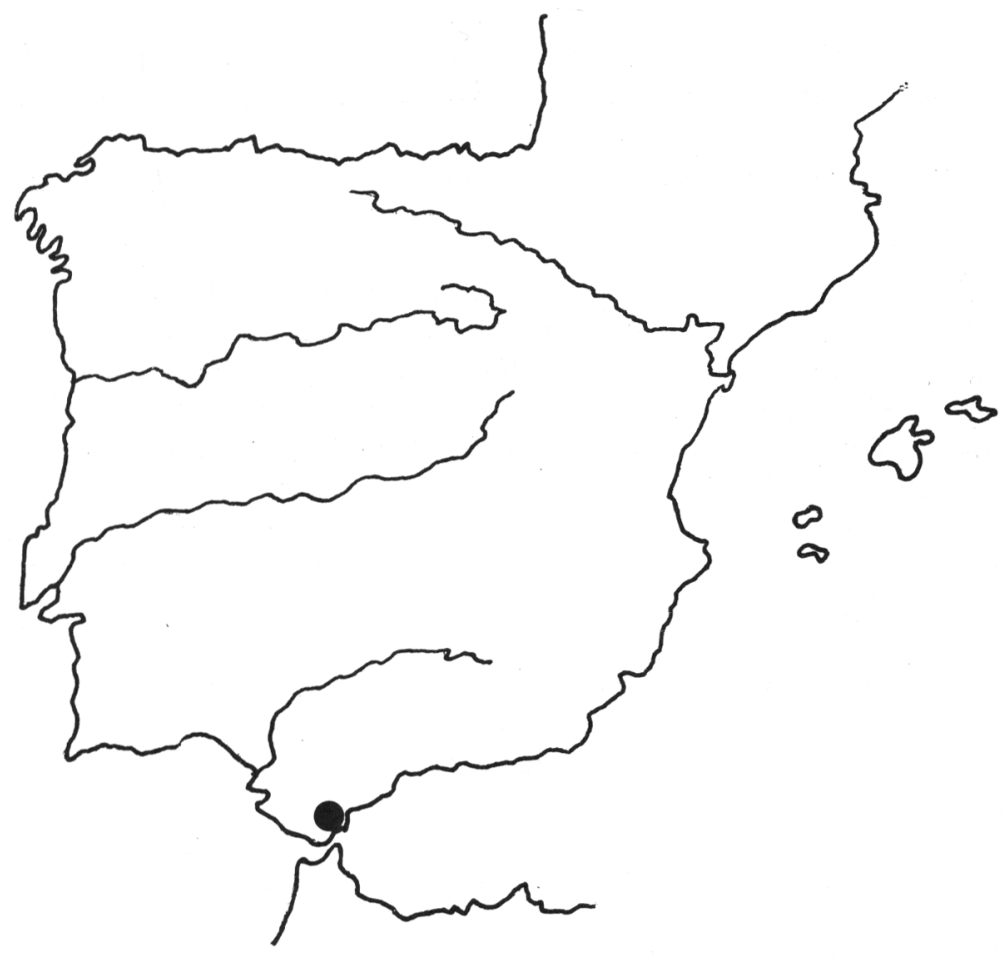

Mapa 1.-Distribución de Polypodium macaronesicum Bobrov en la Península Ibérica 
Polypodium macaronesicum Bobrov, Flory SSSR Bot. Zurn. (Leningrad) 49:534-545 (1965)

P. vulgare L. subsp. azoricum Vasc. in Bol. Soc. Brot. 42: 160 (1968).

P. azoricum (Vasc.) Fernand. in Bol. Soc. Brot. 42: 242 (1968).

P. australe Fée subsp. azoricum (Vasc.) Nardi in Webbia 31 (1): 79-96 (1977).

Páleas del rizoma tenues de 6-13 mm. de longitud, de color amarillo a marrónamarillentas, lanceoladas a ovado-lanceoladas prolongándose en su extremo apical en un largo apéndice filiforme, márgenes denticulado-espinosos muy patentes.

Lámina triangular a ovado-triangular, lo más a menudo tan larga como ancha. Segmentos anchos, obtusos en su extremo distal, atenuándose hacia la base, formando un ángulo de $90^{\circ}$ con el raquis, márgenes serrados o crenados. Soros grandes, orbiculares con numerosísimos esporangios, número de células engrosadas del anillo entre 10 y 14 (frecueńcia máxima de 12), células basales del esporangio (aquellas que unen el anillo de dehiscencia al pedículo del esporangio) $0-1$. Paráfisis raros, poco ramificados, cortos y quebradizos. I-1855).

Isotypus: In muris monasterii San Diego del Monte. Tenerife. (Bourg. 1559. 26-

Distribución: Elemento macaronésico (Canarias, Azores y Madeira) y delSW de Europa (Sierras de Algeciras).

Entre los testimonios existentes en herbarios, podemos destacar los siguientes:

Cádiz: Caño del Agua, Presa del Guadarranque. Castellar de la Frontera, V1973, Asensi et Diez (MGC 1.125) (30 STF 7250); Garganta del Capitán, 2-V-1975, Asensi, Hernández et Diez Garretas (MGC 2.888) (30 STF7602); Valle del Río de la Miel, V-1976, Salvo Tierra (GDA 5.884) (30 STE7699); Nacimiento del Río de la Miel, V-1979, Salvo Tierra (GDA 5.885-6)(30 STE 7.598); Sierra de Ojén, sf., Molesworth (Herb. Molesworth 8.724).

Azores: Ilha do Faial, horta. Alt. 15 m., 16-I-1968, Botelho Gonçalves (exc. LISI 2.086) (MGC 697).

Tenerife: S. Diego del Monte, in muris monasterii, 26-I-1855,(Bourgeau Plantae canariensis, exc. N. ${ }^{\circ}$ 189) (MA 163.707); Los Organos, 29-IV-1935 (Cuatrecasas, exc. n. ${ }^{\circ}$ 514) (MAF 61.946); Monte de las Mercedes, 7-IV-1975, Asensi, Diez Garretas et Antúnez (MGC 2.263).

Gran Canaria: S. Mateo, 18-IV-1935 (Cuatrecasas, exc. n. ${ }^{\text {91) }}$ (MAF 61.947)

Ecología: En el S. de la Península Ibérica, se comporta como terrícola, a veces epífito, acidófilo y umbrófilo; vive sobre suelos desarrollados en las areniscas del Triásico; se le localiza en biotipos de clima atlántico húmedo. Su estación ecológi- 


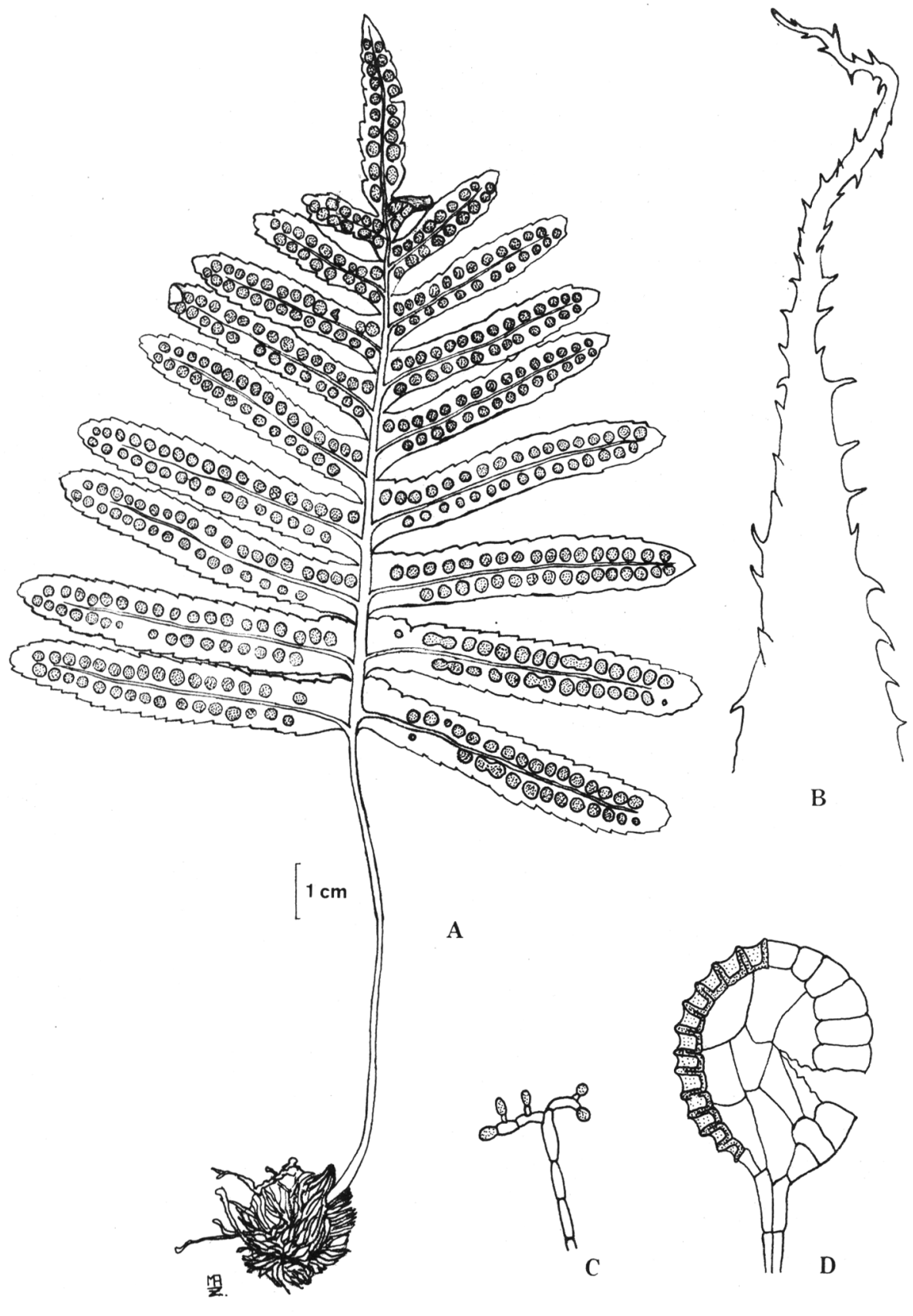

Figura. 1.- Distintos detalles morfológicos de Polypodium macaronesicum Böbrov.: A) Fronde; B) Silueta de escama interna del rizoma (x 9,3); C) Paráfisis (x 93); D) Esporangio (x 93). 
ca preferente son los barrancos o "canutos» de alisos y rododendros (Frangulo-Rhododendretum baetici) si bien en algunas estaciones privilegiadas puede presentarse como epífito de Quercus canariensis (Rusco bippophylli-Quercetum canariensis) donde forma comunidad con Davallia canariensis y un cesped briofítico formado principalmente por Porella canariensis, Neckera cephalonica, Metzgeria furcata, Radula complanata, Orthotrichum lyellii, etc.

Fenología: Para todo el material paninsular, la época fértil comienza en la primavera, mientras que Fernandes $(1968$, b) habla de principios de verano para el inicio del desarrollo en los ejemplares macaronésicos.

\section{CLAVE DE LAS ESPECIES EUROPEAS DEL GENERO POLYPODIUM}

Proponemos como clave de determinación, la publicada por Amaral Franco (1971) basada en los trabajos de Fernandes (1968 a y b) y que nosotros modificamos según los criterios de Nardi (1977), Badré et Prelly (1978) y nuestras observaciones.

1.- Páleas del rizoma de 5 a $13 \mathrm{~mm}$. Hojas con limbo ancho (Indice foliar de 0,8 a 2,0$) \ldots \ldots \ldots \ldots \ldots \ldots 2$

2.- Anillo de dehiscencia con (5-) 6-8 (-9) células engrosadas; soros con abundantes paráfisis; $\mathrm{n}^{\circ}{ }^{\circ}$ de células basales del esporangio $(2-) 3-4 \ldots \ldots$. . australe Fée

2.- Anillo de dehiscencia con 10-13 (-14) células engrosadas; soros con escasos paráfisis, poco ramificados, cortos y fácilmente quebradizos; $n .^{\circ}$ de células basales del esporagio 0-1 (-2)

$$
\text { ........... P. macaronesicum Bobrov }
$$

1.- Páleas del rizoma de 3 a $6 \mathrm{~mm}$. Hojas con limbo estrecho (Indice foliar de 1,5 a 4,0$) \ldots \ldots \ldots \ldots \ldots, \ldots 3$

3.- Soros orbiculares; anillo con más de 10 células de pared engrosada................. vulgare L.

3.- Soros elípticos; anillo con menos de 10 células de pared engrosada ................ P. interjectum Shivas

Observaciones: Sobre aquellos caracteres, a los que Nardi concede un importante valor descriminante, creemos que deben hacerse las siguientes precisiones, de una parte entre el material estudiado hemos encontrado algunos esporangios cuyo número de células del anillo supera la veintena eigualmente otros descienden por debajo de diez, por lo que en nuestra opinión sería deseable recomendar para este tipo de estudios una observación mínima de diez esporangios por soro y de cinco soros por fronde. De otra parte, recomendamos igualmente realizar un número elevado de observaciones de escamas, tanto externas, como internas, ya que en $P$. australe esporádicamente suelen aparecer escamas con dientes largos y densamente repartidos.

Al igual que Nardi, hemos observado que los índices peciolares y foliares en los ejemplares europeos de $P$. macaronesicum, varian bastante, confundiéndose con los de $P$. australe, e igual ocurre a la inversa. Este hecho, según nuestras observacio- 
nes, parece estar ligado a la estación ecológica que una u otra especie colonizan.

Entre los ejemplares estudiados hemos encontrado varios casos que presentaban caracteres comunes de $P$. australe y $P$. macaronesicum, sobre todo en la zona de ecotonía de estas especies, incluso en algunas localidades hemos observado ejemplares que presentaban esporangios abortados, tratándose posiblemente de un híbrido, aún sin determinar, ya que en esta zona, además de $P$. macaronesicum y $P$. australe, hemos localizado $P$. intejectum, cuya presencia no había sido indicada en el Catálogo Florístico de la provincia de Cádiz. (Galiano \& Silvestre, 1974).

Creemos que un fenómeno como éste, concuerda con el señalado porVida \& Reichstein (1975) para las especies europeas del género Polystichum. Estos autores observaron que cuando Polystichum lonchitis (L.) Roth. (alpino-boreal) o P. setiferum (Förskal) Woynar (mediterráneo-pedemontano) crecían en la zona de ecotonía con $P$. aculeatum (L.) Roth. (atlántico-pedemontano) aparecían formas intermedias, no híbridos, imputables a cualquiera de los tres táxones. A dicho fenómeno se le dieron dos posibles interpretaciones: o bien la ductilidad cromosómica del género Polystichum era tan fuerte, que el medio ambiente en que crecía propiciaba una forma fenotípica determinada, o bien, por una introgresión genética, hibridación y posterior retrocruzamiento, se originaban unas formas morfológicamente intermedias.

Por último, queremos exponer algunas consideraciones acerca del origen de $P$. macaronesicum.

Para Manton (1950), la presencia de las tres especies del género Polypodium, hasta ahora citadas en Europa, tienen un origen distinto. Alrededor del globo fue distribuido primariamente un "stock" diploide, el cual se separó en ecoespecies que fueron seleccionadas durante el período glaciar. $P$. australe fue la única especie que sobrevivió en Europa, refugiándose durante las etapas más frías en algunas localidades térmicas, probablemente del norte de Africa, una vez las condiciones fueron favorables inició su difusión por el continente europeo. P. vulgare posiblemente tiene su origen en el extremo oriental de Asia, desde donde emigró, quedando aún allí en algunas localidades relícticas. $P$. intejectum es fruto de la hibridación de $P$. vulgare con $P$. australe y posterior duplicación, apareciendo tras el encuentro de ambos progenitores.

La indudable similitud de $P$. macaronesicum a $P$. australe nos hace pensar en dos posibles explicaciones de su origen:

1.- Ambas especies poseen un ancestro común, tal vez aquel mismo «stock» diploide procedente de la flora tropical. Una de ellas ( $P$. macaronesicum) fue evolucionando dentro de un nicho ecológico termohigrófilo, mientras que la otra $(P$. australe) prefirió aquellas estaciones termoxerófilas. Con la llegada de las glaciaciones, la primera quedó relegada a aquellas localidades donde las condiciones terciarias aún persistían, mientras que la segunda, permanecería durante estos períodos fríos, en localidades no muy influenciadas, aprovechando las etapas interglaciares para extenderse.

2.- Al no encontrarse $P$. australe en el archipiélago macaronésico, posiblemente esta especie sea más moderna que $P$. macaronesicum, quedando esta última 
relegada, durante las glaciaciones, a su actual distribución. Al tener la necesidad de colonizar nuevos nichos ecológicos en los últimos períodos interglaciares tuvo que adaptarse a las exigencias de un clima mediterráneo más xérico, evolucionando perfectamente ante estas nuevas condiciones hasta alcanzar un fenotipo diferenciado de su original precursor.

\author{
BIBLIOGRAFIA
}

AMARAL FRANCO, J. 1971. Nova flora de Portugal, vol. I: 32-33. Lisboa.

ASENSI, A. \& DIEZ, B. 1975. Catálogo florístico de la provincia de Málaga. I. Acta Botánica Malacitana, 1: 7-25. Málaga.

BAADRE, F. \& PRELLI, R. 1978. Les espèces du groupe Polypodium vulgare du Massif armoricain. Candollea, 33 (1): 89-106. Genève.

BELlOT, F. \& CASASECA, B. 1973. Herborizaciones en Canarias. Anal. Inst. Bot. Cavanilles, 30: 109-116. Madrid.

CABEZUDO, B. 1978. Pteridófitas de la provincia de Huelva. Lagascalia, 8 (1): 3-12. Sevilla.

CEBALlOS, L. 1951. Vegetación y Flora Forestal de las Canarias Occidentales. Inst. Forest. Inv. y Experiencias. Madrid.

- 1953. Macaronesia: Consideraciones sobre su flora y vegetación forestal. Conferencia pronunciada en el Inst. Sup. de Agron. de Lisboa. Pub. Esc. Esp. de Ing. Montes. Madrid.

FERnANDES, R. B. 1968a. O género Polypodium L. en Portugal Bol. Soc. Brot. 42: 33-158. Coimbra.

- 1968b. Sobre a ocurrência do complexo Polypodium vulgare nos Açores. Bol. Soc. Brot.. 42: 241-248. Coimbra.

FERNANDEZ LOPEZ, C. 1979. Flora y vegetación del suroeste de la provincia de Jaén. Tesis doctoral. Univ. de Granada.

GALIANO, E. F. \& SILVESTRE, S. 1974. Catálogo de las plantas vasculares de la provincia de Cádiz. I. Pteridophyta-Loranthaceae. Lagascalia 4 (1): 85-119. Sevilla.

- \& VALDES, B. 1971. Catálogo de las plantas vasculares de la provincia de Sevilla. I. Lagascalia, 1: 5-25. Sevilla.

JALAS, J. \& SUOMINEN, J. 1972. Atlas Florae Europeae. I. Pteridophyta. Helsinki.

LOSA ESPAÑA, M. \& RIVAS GODAY, S. 1968. Estudio florístico y geobotánico de la provincia de Almería. Arch. Inst. Aclim., 12 (1). Almería.

MANTON, I. 1950. Problems of cytology and evolution in the Pteridophyta. University Press. Cambridge.

MINISTERIO DE AGRICULTURA. 1970. Mapas comarcales de suelos. Campo de Gibraltar (Cádiz). Madrid.

NARDI, E. 1977. Note sistematiche sul Polypodium australe s.l. della Isole Atalantiche (Azzorre, Madera, Canarie). Webbia 31 (1): 79-96. Firenze.

PINTO DA SILVA, A.R. \& Q. G. 1974. Ferns and flowering plants of the Azores Agronomia Lusit. 36 (2): 5-94.

ROMARIZ, C. 1953. Flora de Ilha da Madeira (Pteridófitos). Rev. Fac. Ciencias Lisboa, 3 (1): 53-112. Lisboa.

RON ALVAREZ, M.E. 1973. Plantas canarias en el Herbario del Jardín Botánico de Madrid. Anal. Inst. Bot. Cavanilles, 30: 117-181. Madrid.

SALVO TIERRA, A. E. 1979. Pteridófitos de Granada. Tesina de Licenciatura. Universidad de Granada. (Inédita). 
SHIVAS, M. G. 1962. The Polypodium vulgare complex. Brit.-Fern. Gaz., 9 (3): 65-70.

SOTA, E. R. 1973. On the classification and phylogeny of the Polypodiaceae. Bot. Journ. Linn. Soc., 67 (1): 229-244.

VASCONCELLOS, J. de CARVALHO 1968a. Nota sobre o polipódio dos Açores. Bol. Soc. Brot., 42: 159-160. Coimbra.

- 1968b. Pteridófitas de Portugal continental e ilhas adjacentes. Ed. Fundaçao C. Gulbenkian. Lisboa.

VIDA, G. \& REICHSTEIN, T. 1975. Taxonomic problems in the fern genus Polystichum caused by hybridization. Europ. Florist. \& Tax. Stud.

WILMANNS, O. \& RASBACH, H. 1973. Observations on the Pteridophytes of Sao Miguel, Açores. Brit. Fern. Gaz., 10 (6): 315-329.

(Recibido el 3 de septiembre de 1979) 